\title{
Mythos
}

MYTHOS

Rivista di Storia delle Religioni

$14 \mid 2020$

Varia

\section{Eracle in Magna Grecia: una porta verso l'eroizzazione?}

Heracles in Magna Graecia: a Gateway to the Heroization?

\section{Flavia Frisone}

\section{(2) OpenEdition \\ 1 Journals}

\section{Edizione digitale}

URL: https://journals.openedition.org/mythos/1961

DOI: $10.4000 /$ mythos. 1961

ISSN: 2037-7746

\section{Editore}

Salvatore Sciascia Editore

\section{Notizia bibliografica digitale}

Flavia Frisone, «Eracle in Magna Grecia: una porta verso l'eroizzazione?», Mythos [Online], 14 | 2020, online dal 31 décembre 2020, consultato il 05 octobre 2021. URL: http://journals.openedition.org/ mythos/1961 ; DOI: https://doi.org/10.4000/mythos.1961

Questo documento è stato generato automaticamente il 5 octobre 2021.

Mythos 


\title{
Eracle in Magna Grecia: una porta verso l'eroizzazione?
}

\author{
Heracles in Magna Graecia: a Gateway to the Heroization?
}

\section{Flavia Frisone}

1 Quando, nel presentare questa relazione e poi nel redigerne il testo avevo rivolto il mio ringraziamento agli organizzatori del doppio appuntamento fra Bologna e Montpellier, mai avrei pensato di dover trasformare questo pensiero nell'estremo saluto e omaggio a uno di loro, Federicomaria Muccioli. Anfitrione dell'incontro bolognese, Federico aveva voluto coinvolgermi in questo convegno in considerazione di alcuni spunti di confronto che avevamo avuto su questo tema, a cui lavorava intensamente da alcuni anni. Alla sua memoria, con grande rispetto e amicizia, voglio perciò offrire la mia dedica particolare all'interno di questa raccolta di studi a cui egli teneva molto, che ha curato fino alla fine e che ora ne ricorda la figura.

2 Il convegno bolognese, con il suo "gemello" di Montpellier, poneva al centro della riflessione i complessi percorsi culturali e religiosi che consentono la definizione di uno statuto superumano, sia esso eroico o divino, nel mondo greco dall'età arcaica a quella ellenistica. Nell'ambito di quest'orizzonte ancora aperto e in gran parte da esplorare, il mio contributo approfondisce quello che può a buon diritto definirsi un topos del panorama storico-religioso della grecità d'Occidente: la figura di Eracle, di cui si osserveranno alcuni aspetti in riferimento all'ambiente magnogreco di età arcaica $\mathrm{e}$ classica ${ }^{1}$.

3 I segni di questo culto fra i Greci dell'Italia, già registrati sistematicamente a partire dai benemeriti studi del Giannelli2 ${ }^{2}$, nel primo quarto del secolo scorso, hanno consentito approfondimenti illuminanti, tali da mettere in luce la remota antichità a cui risalgono gli elementi essenziali del profilo mitico-religioso di questa figura divina già per i primi gruppi coloniali e, da qui, all'interno dei sistemi cultuali di riferimento delle poleis magnogreche. È questo il caso, in particolare, dell'ambiente acheo-coloniale, in cui gli studi di M. Giangiulio, a partire da Crotone, hanno evidenziato la coerenza profonda del peculiare rapporto fra Eracle ed Hera, la divinità di riferimento di tutte le comunità achee d'Italia meridionale, certo presso i famosi Heraia del Lacinio e del Sele ma 
adombrabile anche per i culti di Sibari e Metaponto ${ }^{3}$. Secondo lo studioso, infatti, la documentazione, costituita essenzialmente dai racconti mitici, rielaborati in sede locale, lascia emergere una solidarietà profonda, originaria, fra le figure religiose dell'eroe e della dea, le cui radici affondano nelle tradizioni dell'ambiente metropolitano. Le forme rituali, poi, lasciano intuire un'architettura articolata di culti gerarchicamente destinati e ordinati o, addirittura, nel caso del santuario Lacinio di Crotone, una peculiare «struttura 'bipolare'» fra santuario e città, come fra dea ed eroe $^{4}$. In altri contesti, invece, è stato osservata la creazione di un vero e proprio paesaggio cultuale, nel quale i cicli narrativi 'per immagini' che decoravano gli edifici sacri, di cui sono esempio straordinario - ma certo non caso unico - le metope dell'Heraion del Sele ${ }^{5}$, selezionavano fra le imprese dell'eroe quelle più appropriate al contesto locale e ai significati che esso riteneva di veicolare ${ }^{6}$, costituendo un ulteriore passaggio del radicamento di Eracle nel panorama religioso locale.

4 L'incidenza di Eracle negli ambienti coloniali è inoltre un fattore per misurare la diffusione panellenica (ed extra-ellenica) delle complesse vicende mitiche dell'eroe, plasmate e rifunzionalizzate in diversi contesti storici e geografici non solo e non tanto nella dimensione "alta" dell'epos, quanto, come è stato osservato ${ }^{7}$, in quella più accessibile e "popolare" che si riflette nelle forme e nei temi di un poliedrico repertorio figurativo ${ }^{8}$. In questa prospettiva, anzi, l'ambiente coloniale non solo consente di apprezzare l'intrinseca capacità di questa figura religiosa di farsi portatrice di istanze di livello diverso - da quello personale a quello collettivo e politico - ma dimostra di essere stato uno dei poli attivi nello sviluppo dei temi mitico-narrativi che la coinvolgono. Essi ben si prestano, del resto, a raccordare l'esperienza delle nuove comunità elleniche all'identità culturale del più vasto "mondo greco", legandone insieme la complicata fisionomia.

5 Il radicamento locale di vicende mitiche e culti eracleici fra le comunità elleniche di Sicilia e Magna Grecia, tuttavia, non sempre è agevole da cogliersi nella sua giusta luce. È ben nota l'enfasi con cui, più che il valore religioso, se ne è sottolineata la funzione nel quadro dei rapporti d'interazione nel contesto coloniale. Sui limiti di questo approccio interpretativo ho avuto modo di riflettere altrove, e in particolare in uno studio recente centrato sulla figura e sul culto di Eracle nella Sicilia greca, a cui rimando per le osservazioni relative proprio a questi aspetti, che non sarà quindi necessario riprendere in dettaglio in questa sede ${ }^{9}$. Dal punto di vista religioso, invece, la dimensione "relazionale" di Eracle nei confronti di ambiti culturali differenti da quello greco appare un elemento importante nello scenario magnogreco: in questo culto, del resto, si esemplifica uno dei casi più appariscenti di collegamento fra le forme religiose di origine ellenica e quelle radicatesi nella prassi cultuale e nell'ideologia religiosa italica $^{10}$. La consistenza e diffusione dei culti eracleici presso i diversi interlocutori greci con cui le differenti popolazioni italiche s'interfacciano - anche se alcune, come gli Etruschi e i Romani, risentono certamente di più vasti circuiti trans-mediterranei, in cui fondamentale appare l'apporto fenicio ${ }^{11}$ - sembrerebbe fondata ex silentio su questa osservazione. Un vero riscontro, tuttavia, non può prescindere da una messa a fuoco dell'effettiva incidenza dei culti di Eracle presso le singole comunità elleniche, che tenga conto del quadro documentario aggiornato alle più recenti indagini sulle poleis magnogreche e sui loro territori ${ }^{12}$. A questo obiettivo ha voluto rispondere la presente ricerca, ponendosi in continuità con il lavoro, sopra citato, portato a termine per la Sicilia. La discussione dei risultati di questa sezione, purtroppo, eccede lo spazio consentitomi per questo contributo e, dal momento che essa appare autonoma rispetto 
al tema che specificamente interessa in questo incontro, ho preferito destinarne la pubblicazione in un momento successivo, in questa medesima sede editoriale ${ }^{13}$.

6 Ai fini del nostro assunto, invece, si seguiranno gli spunti e le suggestioni offerti dalla tradizione mitico-narrativa intorno alla presenza di Eracle in Magna Grecia - tradizione consistente e articolata, nonché, come si è detto, a più riprese oggetto di studi di fondamentale interesse.

7 Come si sa, il filo rosso che lega queste tradizioni relative al passaggio dell'eroe in Occidente, e in particolare nei territori che saranno dei Greci d'Italia, pur nei diversi contesti genetici in cui è possibile contestualizzarli, è il loro impianto 'storicizzante'. Essi offrivano cioè la possibilità di rendere intelligibile un tempo delle origini, mettendolo in relazione "cronologica" (i.e. mitico-genealogica) con il passato non indistinto in cui si muove l'eroe, al tempo stesso in cui davano conto, rispetto al presente, dei caratteri naturali e antropici dei differenti territori in cui le comunità locali si riconoscevano, trasformandoli in paesaggi culturali loro peculiari. In questa chiave, i miti di Eracle assumono così la duplice funzione di precedenza e di eziologia, in relazione ai "segni" tangibili attribuiti al passaggio del dio: istituzioni e culti da lui fondati ma anche mirabilia legati alle sue gesta ${ }^{14}$.

8 Com'è stato ben messo in luce dalla critica, una cornice stabile e autorevole per l'inquadramento ma anche per lo sviluppo di tradizioni mitiche era rappresentata dalla rilettura stesicorea del viaggio di ritorno dall'impresa gerionica ${ }^{15}$, quella che sarà codificata come decima fatica dell'eroe. Questa infatti fornisce alla struttura narrativa una forma itineraria e diegetica che verrà accolta e cristallizzata dalla tradizione posterior $\mathrm{e}^{16}$, con la possibilità di aggiungervi elaborazioni successive e connessioni secondarie e collaterali, di ambito locale.

9 In questo ampio repertorio sono stati intravisti tre foci principali delle imprese di Eracle in Italia meridionale. Da una parte infatti c'è l'area campana, o più precisamente Cuma, cui si lega, accanto e forse ancor più che il ricordo del percorso dell'eroe con le vacche sottratte al mostro tricorpore Gerione, il suo ruolo nella lotta contro i Giganti ${ }^{17}$. Questa addirittura, nella versione occidentale che, per esplicita indicazione di Diodoro Siculo, sappiamo essere seguita da Timeo ${ }^{18}$, diviene, da scontro cosmico di cui sono protagonisti Zeus e gli Olimpii, una lotta di Eracle contro i fortissimi esseri nati dalla terra, in cui sono gli dei ad avere la funzione di symmachoi al fianco dell'eroe ${ }^{19}$. Ricollocata nello scenario di una Phlegra ora posta nella pianura campana, la vicenda mitica viene agganciata a localizzazioni (per esempio dei luoghi di supplizio dei mostruosi avversari sconfitti dagli dei olimpi) ${ }^{20}$ che sono state viste come una ricodificazione ambientale di matrice culturale coloniale euboico-calcidese ${ }^{21}$.

La medesima componente etnico-culturale elabora, nelle sedi siceliote e a Rhegion, le vicende che inquadrano l'estrema punta meridionale della Calabria e l'area dello Stretto di Messina. Qui i miti eracleici da un lato segnano la connessione con la Sicilia focalizzando, con versioni differenti, il passaggio della mandria sacra nell'isola e la complicata ricerca da parte dell'eroe, dall'altro lasciano emergere un immaginario più antico legato alle prime frequentazioni del porthmos da parte dell'eroe la cui figura religiosa rende pervio e intellegibile agli uomini lo spazio ignoto ed accompagna l'elaborazione della minaccia di un pericolo estremo ${ }^{22}$.

11 Un ultimo cluster di vicende mitiche si raccoglie nell'area ionica della Calabria, trovando in Locri e soprattutto in Crotone e nel suo territorio interno i nodi principali, cui si giustappongono altre tappe (Eraclea, Metaponto) ${ }^{23}$. È degno di nota che proprio in 
questo circuito di poleis una significativa elaborazione della figura di Eracle - in termini strettamente correlati alla costruzione identitaria degli insediamenti e ai rapporti inter-poleici - lasci parallelamente traccia di sé nell'ambito del linguaggio della comunicazione istituzionale, e segnatamente nella scelta dei tipi monetali, fenomeno analizzato in dettaglio nella seconda parte di questa ricerca, dedicata alla documentazione primaria ${ }^{24}$. Non sorprende dunque che proprio qui si concentri una serie di tradizioni che, pur riproponendo schemi tipici delle narrazioni eracleiche, si lasciano individuare per alcuni peculiari elementi e sviluppi ${ }^{25}$. Da un lato, infatti, vi si osserva il ripetersi e sovrapporsi di racconti mitici che s'impostano sul topos del conflitto violento fra l'eroe e una serie di avversari aggressivi e anomici, schema che caratterizza generalmente la sua vicenda occidentale ${ }^{26}$, dall'altro emerge qui una forte valenza eziologica, esplicitamente connessa alla fondazione di culti ovvero alla legittimazione di una relazione privilegiata dell'eroe con le comunità coinvolte.

Spiccano in primo luogo le tradizioni relative a Crotone, che inquadrano sia l'origine della città che quella del santuario di Hera al Lacinio. Racconti analoghi si tramandavano anche per Locri e per Metaponto, sebbene in quest'ultimo caso nella formulazione controversa di una fonte tarda ${ }^{27}$, mentre ad Heraklea il mito assumeva un profilo specifico.

13 È Diodoro Siculo che enuclea, in riferimento a Crotone, l'essenziale traccia narrativa. Di seguito al racconto della deviazione in Sicilia del viaggio di Eracle di ritorno dalle terre dell'Iberia, l'Agirineo narra come questi, ritornato sul continente con le sue splendide vacche, prosegua il suo viaggio lungo la paralia dell'Italia. Anche in queste lande egli deve sventare un ennesimo tentativo di sottrargliele e in un tale frangente uccide involontariamente Crotone, suo ospite coinvolto per errore nel tentativo di furto da parte del suocero Lacinio. Come compensazione del destino infelice del suo giusto amico, Eracle ne cura un rituale funebre $\mu \varepsilon \gamma \alpha \lambda$ eroica con la profezia che in futuro su queste stesse terre sorgerà una città che porterà il nome di quello ${ }^{28}$.

14 La tradizione riferita dall'autore di età cesariana, che potrebbe averla ripresa da Timeo $^{29}$, citato più volte poco prima di questo passaggio, sembra però essere più antica. Infatti proprio a questo tema - come unanimemente ritiene la critica, sebbene con sfumature interpretative differenti ${ }^{30}$ - rimandano le coniazioni argentee emesse da Crotone sia negli anni Venti del V secolo sia successivamente ${ }^{31}$. In particolare la serie più antica, recante sul $\mathrm{D} /$ un Eracle nudo assiso e legenda OIKISTAS ${ }^{32}$, volutamente arcaizzante nei caratteri alfabetici ${ }^{33}$, ci consente di risalire almeno fino a circa il 425 a. C. D'altro canto, la testimonianza di Giamblico, che si rifà a fonti pitagoriche che rimontano almeno al periodo fra $\mathrm{V}$ e $\mathrm{IV}^{34}$, lascerebbe pensare che la leggenda di un coinvolgimento di Eracle nella fondazione fosse nota e condivisa già nella seconda metà del VI secolo, in quanto Pitagora, nell'ambito del primo logos da lui pronunciato una volta giunto presso i Crotoniati, vi avrebbe fatto riferimento in termini, come vedremo, particolarmente significativi.

Alcuni riflessi di una ripresa di questo filone mitico - o meglio di un suo articolarsi alla luce dello stringente rapporto di Eracle con il santuario Lacinio e della centralità di questo per la polis di Crotone - si colgono in riferimento a una notizia tradita da Servio, secondo cui Eracle è da taluni ritenuto fondatore del culto in onore della dea ${ }^{35}$. Anche in questo caso la relazione trova una sua specifica visibilità nelle emissioni monetali ${ }^{36}$, il che consente di definire un terminus di fine $\mathrm{V}$ o dei primi anni del IV, mentre $\mathrm{i}$ 
riecheggiamenti in altre fonti secondarie mostrano che confusioni e varianti si erano stratificate nel tempo sul medesimo tema ${ }^{37}$. Alcuni degli stessi elementi (onomastica dei personaggi, e relazioni fra loro, riferimenti a luoghi) ricorrono in una notizia riferita a Locri ma tanto simile alla precedente da essere a lungo stata guardata con sospetto ${ }^{38}$. In base ad essa un'analoga profezia di fondazione da parte di Eracle, a seguito dell'omicidio involontario di un Locro, fratello di Alcinoo, proveniente dalla Feacia, dopo un'uccisione che ricalca in molti dei minimi dettagli quella di Crotone, veniva posta all'origine di Locri Epizefirii ${ }^{39}$.

Qualche studioso ha voluto far risalire l'origine prima di questa notizia, e il contesto storico della sua genesi, a un gioco di opposte "propagande" fra Locresi e Crotoniati al tempo della battaglia della Sagra, riferimento che ci porterebbe indietro anche rispetto all'orizzonte pitagorico della notizia di Giamblico su Crotone ${ }^{40}$. Devo confessare che l'ipotesi non mi sembra convincente e che trovo più opportuno registrare il dato minimo che da queste fonti si può ottenere: vale a dire l'esistenza di tradizioni mitiche ricorrenti - ma non necessariamente alternative o concorrenti - che fanno leva sulla figura di Eracle, personaggio rilevante nel paesaggio cultuale di entrambe le poleis, per legittimare un eroe eponimo al quale in nessuno dei due contesti può essere attribuita la qualità di ecista, ma al quale probabilmente si assegna un culto di tipo eroico.

Questo processo, che comporta l'innestarsi del patrimonio mitico - rinnovato attraverso l'istituzione (più che probabile) di rituali religiosi di valenza locale ${ }^{41}-$ nel tessuto vivo delle vicende storiche dell'Arco Ionico, è stato messo in evidenza brillantemente da M. Nafissi per Eraclea, la colonia tarantina stabilita nel 433/2 a. C. in vece della fondazione mista thurino-tarentina, che aveva fatto risorgere l'antica fondazione di Siris. Allo studioso si deve infatti l'indagine esaustiva della complessa tradizione che, impostandosi sul medesimo filone eracleico, confluisce nella testimonianza dell'Alessandra di Licofrone ${ }^{42}$. La vicenda mitica rievoca un Calcante Sisifeo ucciso da Eracle per i "mal contati fichi" la cui tomba eroica e il culto fondato da Eracle sulle sponde del Siris vedranno un giorno rinnovarsi l'insediamento di molti abitanti: secondo l'illuminante esegesi del Nafissi l'elaborazione, in termini tanto mitico-religiosi quanto politici, di un «precedente mitico della fondazione di Herakleia» ${ }^{43}$.

Proprio l'efficace chiarimento di questa criptica testimonianza da parte del Nafissi ci consente di acquisire un ulteriore elemento utile al nostro assunto. In primo luogo esso ci riporta saldamente entro una cornice storica: in un orizzonte che può porsi almeno nella seconda metà del $\mathrm{V}$ secolo a. C. la figura religiosa di Eracle è un elemento attivo per l'istituzione di rituali e per la loro legittimazione, anche in chiave politicodiplomatica. Come giustamente osserva lo studioso è probabile che si possa intravedere dietro la notizia elaborata dalla fonte poetica - e nel suo contesto storico - la concreta istituzione di un culto eroico di Calcante in cui il legame con Eracle, essenziale a livello mitico, doveva essere espresso in forma rituale anche con la relazione al culto, "gerarchicamente" superiore, di quello, il cui carattere doveva essere divino. Non diversamente la relazione appare posta in un'altra importante testimonianza, quella di Diodoro Siculo per Agirio, dove viene fondato da Eracle un duplice culto eroico, per Gerione e per Iolao, ma nel quadro degli onori divini che gli Agirinei si vantavano di essere stati i primi a concedere all'eroe ${ }^{44}$. In questa prospettiva, dunque, la figura di un Eracle "pienamente dio" appare funzionale alla legittimazione di un culto di statuto eroico: e se culti, agoni e santuari fondati dall'eroe "civilizzatore" per altre divinità non 
sembrano essere una novità, quel che appare collegare questi racconti mitici è il conseguimento di un culto eroico che nei casi in questione passa attraverso la morte ottenuta per mano di Eracle ${ }^{45}$. Ma, se il motivo dell'omicidio (involontario o preterintenzionale) appare necessario per veicolare sul piano mitico l'idea di una netta discontinuità o addirittura una frattura negli assetti locali, occorre rilevare che esso non viene riproposto obbligatoriamente: a Metaponto, terra un tempo del re Alibàs/ Alybas, l'eroe eponimo, suo figlio Metabos, riceve alla nascita il nome che sarà un giorno quello della città, dato in onore di Eracle e del suo passaggio nella casa del

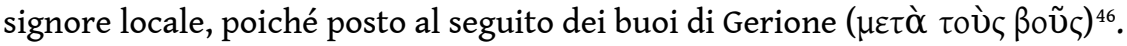

19 A questa temperie spirituale e prassi cultuale che riconosce in Eracle un anello fondamentale per incardinare legittimamente nuovi culti nella realtà locale, potrebbe riconnetterci un altro contesto magnogreco, documentario - e quindi meno esplicito delle fonti precedenti - ma assai conosciuto e discusso nel quadro della problematica dedicata ai culti di ecisti nelle poleis coloniali. Si tratta del monumento meglio noto come heroon dall' agorà di Poseidonia ${ }^{47}$ : l'attestazione archeologica di un culto particolarmente significativo per la colonia achea (tanto da aver sede nel cuore dello spazio da cui si dipana la struttura urbanistica e religiosa della città fin dalle sue origini $^{48}$ ma che appare esser stato diversamente destinato e funzionalizzato ${ }^{49}$, nel corso del tempo, non necessariamente sempre per un ecista e forse per più d'uno ${ }^{50}$. Come si sa, l'edificio con copertura a doppio spiovente che costituiva il cuore nascosto della struttura un tempo visibile, realizzata come un grande tumulo su uno zoccolo di basamento, non conteneva resti funebri ma ospitava un corredo organicamente composto, di cui facevano parte arredi ormai perduti, come una tavola in prezioso legno di cipress ${ }^{51}$ e oggetti selezionati di alto significato simbolico, come un set di spiedi originariamente avvolti in bende, otto vasi in bronzo di finissima fattura e oggetti in ceramica, disposti sui lati lunghi della camera in maniera speculare e contenenti miele in favi. Fra questi due anfore, di cui una, quella collocata nell'angolo $\mathrm{NE}$, di tipo panatenaico a figure nere e con il piede restaurato già in antico, presenta la raffigurazione dell'apotheosis di Eracle ${ }^{52}$.

Ritenuto inizialmente un luogo di culto dedicato alle ninfe, sulla base di testimonianze epigrafiche poi risultate non pertinenti dal punto di vista stratigrafico $0^{53}$, il sacello è stato successivamente riconosciuto come un heroon, possibilmente collegato con il culto dell'ecista $^{54}$, realizzato sullo scorcio del VI secolo e successivamente rimaneggiato in relazione alla sistemazione architettonico-urbanistica dell'agorà. Tralasciando qui, per motivi di opportunità, la questione se si tratti effettivamente del culto civico dell'ecista e a quale ecista si debba pensare ${ }^{55}$, concentriamoci su quel segno di Eracle, l'immagine dell'arrivo del nuovo dio, sul carro guidato da Atena, nell'olimpo in cui lo accolgono altri dei come Apollo, Hermes ed Artemide. L'anfora che la reca è giustamente ritenuta una chiave essenziale per decodificare il contesto archeologico come luogo di culto eroico. Essa deve essere considerata un elemento del contesto sacrale: quell'oggetto e solo quello - a costo di doverlo riparare dopo una frattura ${ }^{56}$ - doveva sembrare appropriato per quella deposizione rituale. La riparazione sul piede, che certo non possiamo attribuire a motivi di economia, indica la necessità di preservare per l'uso un oggetto importante, già impiegato in precedenza, non sappiamo se per il medesimo scopo. La rappresentazione di apotheosis, sulla metopa dipinta, appartiene allo schema più antico del repertorio iconografico dell'assunzione di Eracle fra gli dei, quella che lo vede sul carro condotto da Atena e circondato da altre divinità ${ }^{57}$. Esso focalizza del percorso mitico di Eracle, com'è stato osservato in maniera illuminante, l'“ingresso" 
nella condizione divina distinto e in qualche modo cristallizzato rispetto all'immagine della morte sulla pira, che inquadra piuttosto, in certo senso in maniera polare, l'uscita dai pathea e dalla condizione mortale ${ }^{58}$. Colpisce allora che anche in questo caso un contesto cultuale di rilevante significato civico - sia che si tratti davvero del culto dell'ecista sia che invece si rivolga a una figura che, pur non potendo essere propriamente definita fondatore della polis, si colleghi alla sua origine e alla sua identità, o alla riaffermazione di queste - si definisca nel segno della divinità di Eracle ${ }^{59}$.

Possiamo dunque chiederci, a questo punto, se non esista un nesso profondo ed essenziale fra l'accesso al divino dell'heros-theos e quello allo statuto eroico di chi attraverso lui ottiene venerazione e culti. Un collegamento che fa di Eracle una "porta verso il sacro" in una dimensione cultuale e rituale capace di reinterpretare, confermare e legittimare.

L'esame più approfondito di una delle fonti sopra ricordate a proposito della tomba eroica istituita da Eracle per Crotone potrebbe forse aiutarci ad aggiungere elementi per una risposta. Si tratta dell'episodio della vita pitagorica riferito da Giamblico che vede il filosofo, da poco giunto nella città achea d'Italia, allacciare i primi proficui rapporti con l'aristocrazia dominante (Iambl. v. Pyth. 9, 50):

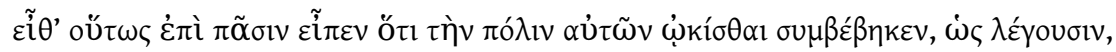

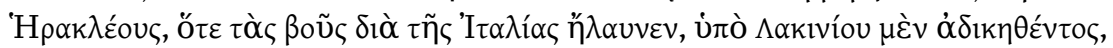

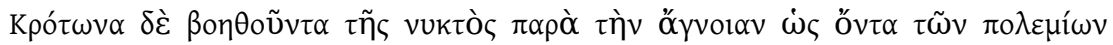

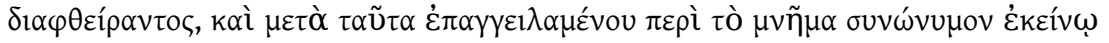

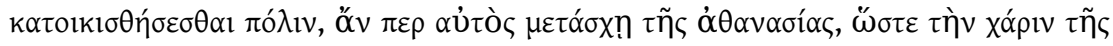

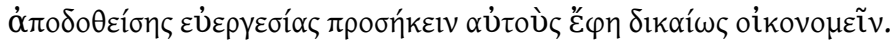

Come si è accennato, la notizia va contestualizzata nella prima predicazione di Pitagora al suo arrivo a Crotone, e in relazione a questo appare coerente che essa mostri di voler radicare la teoresi politico-religiosa introdotta dal filosofo nella tradizione religiosa locale.$^{60}$ In maniera del tutto conforme a ciò, il discorso del Samio cominciava col porre i Crotoniati sotto il nume tutelare di Eracle, a cui attribuisce la dignità di ecista, narrando l'episodio, sopra illustrato, della morte accidentale dell'eponimo. La profezia che una città dal nome di Crotone sarebbe sorta un giorno assume qui la forma di un impegno condizionato all'assunzione da parte di Eracle stesso di una condizione divina. Nella dialettica che il racconto mitico sottende la gerarchia di "valore" religioso appare chiara e comprensibile, assegnando a ciascuno la timé appropriata alle proprie benemerenze: la dignità eroica (e il probabile culto ad essa collegato) spettava all'eponimo (così come - correlatamente - all'ecista) in quanto Eracle era di fatto giunto alla dignità divina e alla vita immortale (l'athanasia), che gli doveva essere riconosciuta nel culto. La "catena" mitico-rituale che lega Hera del Lacinio, Eracle e la città che prende il nome da Crotone implica perciò che a questi, ultimo ed essenziale anello, sia dedicato onore $\mathrm{e}$ culto.

Gli stessi concetti - la familiarità del rapporto con Eracle, la natura divina di questi, l'istituzione di culti correlati a questa condizione - tornano, del resto, anche un altro passo della Vita Pitagorica (Iambl. v. Pyth. 8,40):

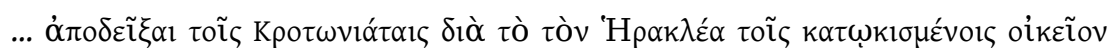

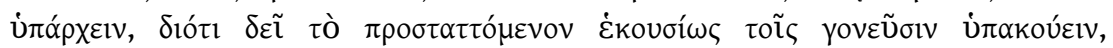

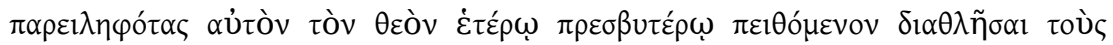

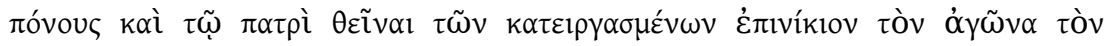
'Òúpurıv. 
26

Anche con questo discorso siamo alla prima allocuzione di Pitagora ai giovani nel ginnasio di Crotone, quindi in un contesto in cui la figura religiosa di Eracle appare centrale, sia in virtù della tradizione cittadina sia per il peculiare legame dell'eroe con le esperienze dell'educazione giovanile; in più, appare cruciale, qui, il riferimento alle prove agonali, al loro significato e valore.

Il pensiero mitico-religioso pitagorico mostra la sua coerenza ma anche le differenze con l'opinione vulgata: gli athla, cui Eracle si sottopone volontariamente per il rispetto dovuto a chi gli è maggiore, non sono la condizione per raggiungere la dimensione divina, ma una manifestazione del suo statuto che è già superumano, celebrato come epinicio dagli agoni olimpici da lui istituiti in onore del padre Zeus. Nuovo e significativo appare anche, come ha ben sottolineato Detienne ${ }^{61}$, l'esplicito inquadramento di Eracle come agente di una euergesìa che deve ispirare giusti comportamenti. Agli occhi dei Crotoniati l'eroe, oltre che figura intrinsecamente divina

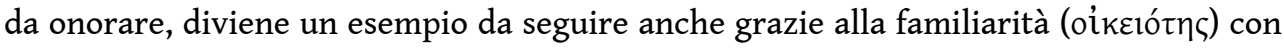
lui, che ispirerà agli adulti la giustizia, ai giovani l'obbedienza.

Può dirsi, in sintesi, che in questa elaborazione pitagorica della figura di Eracle si fondano elementi tipici della sensibilità greca arcaica e spunti innovavi. Da un lato c'è, nel segno della continuità, l'imitatio di dei ed eroi che s'inscrive in modalità comportamentali molto concrete, tipiche del periodo arcaico, di cui non mancano eclatanti esempi sia nel mondo coloniale che metropolitano. Basti ricordare, proprio a Crotone, il caso di Milone, agonista, olimpionico e figura di riferimento della polis che assume la tipologia fisica (il vigore straordinario) e l'apparenza (la leonté, la clava) di Eracle per incarnare una dignità e un valore superiore che aspira a raggiungere uno statuto sovrumano ${ }^{62}$. Anche in altri casi il gioco di risonanze avrebbe consentito di alludere a una condizione di potere e di timè superiore, come nel caso di Pisistrato del famoso racconto erodoteo della sua seconda presa di potere tirannico ${ }^{63}$.

Nella direzione del nuovo, invece, a questa meccanica giustapposizione si sovrimposta una sorta di percorso "didattico" dell'elevazione, che presuppone la possibilità di imparare a praticare la giustizia degli dei tramite la familiarità con loro. Un'innovazione che potrebbe aver avuto sviluppi ben al di là delle premesse, se è vero che essa apre la strada a quello spostamento verso una dimensione metaforica e iniziatica dell'esperienza di $\mathrm{Eracle}^{64}$, fra cui quella che, grazie all'elaborazione del dionisismo italiota, lo fa divenire modello dell'esperienza del "diventare un dio" ${ }^{65}$. Ma con questo immaginario, che sta alla base della produzione dei pittori proto-apuli e proto-lucani e motiva la committenza dei loro grandi vasi funerari ${ }^{66}$ siamo tuttavia su un altro livello, meno aristocratico e certamente meno politico, di quello che ha visto operare il nume salvifico di Eracle in ambito pitagorico.

\section{Conclusioni}

A conclusione di questo excursus sulle tradizioni mitico-religiose di Eracle in Magna Grecia gioverà ripartire da una delle prime considerazioni sopra avanzate: la capacità plastica di questo culto, divino e istituzionalizzato, che tuttavia è in grado di adattarsi a prospettive locali, di "incarnarsi" in esse e prendervi forma ${ }^{67}$. Di ciò sono espressione anche gli sviluppi narrativi del mito che lo collegano ad altri culti divini (Hera soprattutto, in ambito acheo) ovvero ad altre figure del panorama mitico-religioso 
panellenico (p.es. Filottete nella Sibaritide e nella Crotoniatide) o locale (Crotone, Locro, Metabos, Calcante a Siri). Le potenzialità mitopoietiche del personaggio e la dimensione eminentemente itinerante dei suoi miti lo rendono, insomma - lo si è detto - particolarmente efficace nella creazione ed espressione di quel che la moderna terminologia sociologica definisce "località" e "vicinati", cioè quell'insieme di fenomeni che manifestano i legami fra gruppi e comunità definiti in termini di relazioni sociali e contesti spaziali ${ }^{68}$. In sostanza, egli continua a raccontare, a spiegare, a dare supporto e fondamento ai passaggi, anche traumatici, di una storia in divenire, come quella delle città magnogreche. Lo illustra il caso di Eraclea, dove la sua presenza consolida nuove istituzioni e trasforma assetti preesistenti traducendo in termini cultuali il netto cambiamento ma diviene anche - come suggerisce la documentazione numismatica - il "segno" visibile di una dialettica con le tradizioni locali preesistenti.

Parallelamente a ciò, le tradizioni mitiche e i dati documentari esaminati ci mostrano anche che, in un orizzonte storico definito, di età classica, o al più del maturo arcaismo, la figura di Eracle ricorre come elemento attivo a livello culturale e religioso in un ambito che potremmo definire "produzione di sacralità": essa legittima la presenza di nuovo eroe e gli "assegna" un culto, collegato ma gerarchicamente ordinato a quello, divino, rivolto ad Eracle stesso. In alcuni di questi casi, anzi, solo la morte per mano dell'heros-theos è il tramite per il raggiungimento di uno statuto eroico, con la fondazione di un contesto rituale (la tomba) e il suo inserimento nella pratica di culto locale.

Proprio nella misura in cui l'eroe-dio appare un attore importante e ricorrente nello scenario miti-storico regionale, sembra significativo, dunque, che alcuni aspetti peculiari della sua figura religiosa si colleghino a processi di mutamento/metamorfosi verso una condizione sacralizzata, lasciando immaginare di essere parte dei percorsi di acquisizione di tale dimensione. L'Eracle venerato dalle comunità dell'Italia si mostra, in ciò, un fondamentale tramite - divino - del percorso che porta altre figure allo statuto eroico, e appare, anche per questo, figura di riferimento di ideologie che implicano la possibilità di una trasformazione nella direzione del superumano, poiché dà spazio e strumenti culturali all'attuarsi di processi di "divinizzazione".

\section{BIBLIOGRAFIA}

ADORNATO 2003: G. Adornato, «L'area sacra di S. Omobono. Per una revisione della documentazione archeologica», in Mélanges de l'École française de Rome. Antiquité, 115, 2. 2003, 809-835.

Ampolo 2017: C. Ampolo, «Demarato di Corinto 'bacchiade' tra Grecia, Etruria e Roma: rappresentazione e realtà fonti, funzione dei racconti, integrazione di genti e culture, mobilità sociale arcaica», Aristonothos 13.2 (2017 [2018]), 25-134.

APPADURAI 1996: A. Appadurai, Modernità in polvere (ed. or. Univ. of Minnesota Press 1996) tr. it. Milano 2012. 
ARDovino 1986: A. M. Ardovino, I culti di Paestum antica e del suo territorio, Salerno 1986.

ARENA 1996: R. Arena, Iscrizioni greche arcaiche di Sicilia e Magna Grecia. I. Iscrizioni di Megara Hyblaea e Selinunte, Pisa 1996.

BAYET 1926: J. Bayet, Les origines de l'Hercule romain, Paris 1926.

BERNARDINI, ZUCCA 2005: P. Bernardini, R. Zucca (edd.), Il Mediterraneo di Herakles: studi e ricerche, Roma 2005.

BOARDMAN 1975: J. Boardman,

«Herakles, Peisistratos and Eleusis», Journal of Hellenic Studies, 95 (1975), 1-12.

BOARDMAN 1989: J. Boardman,

«Herakles, Peisistratos and the Unconvinced», Journal of Hellenic Studies, 109 (1989), 158-9.

BOARDMAN ET ALII 1988: J. Boardman, O. Palagia, S. Woodford, s.v. Herakles, LIMC IV.1, ZürichMünchen 1988, 728-838.

BoARDMAN ET ALII 1990: J. Boardman, O. Palagia, S. Woodford, W. Felten, G. Kokkorou-Alewras, L. Todisco, P. Brize, V. Smallwood, A.F. Laurens, s.v. Herakles, LIMC V.1, Zürich- München 1990, 1-192.

BONNET 1988: C. Bonnet, Melqart. Cultes et mythes de l'Héraclès tyrien en Méditerranée, Leuven-Namur 1988.

BONNET 2005: C. Bonnet, «Melqart in Occidente. Percorsi di appropriazione e di acculturazione», in P. Bernardini, R. Zucca (edd.), Il Mediterraneo di Herakles: studi e ricerche, Roma 2005, 17-28.

BONNET 2008: c. Bonnet, «Le tradizioni eraclee nella Calabria tirrenica», in G. De Sensi Sestito (ed.), La Calabria tirrenica nell'antichità. Nuovi documenti e problematiche storiche, Soveria Mannelli 2008, 343-352.

BONNET, JOURDAIN-ANNEQUIN 1992: C. Bonnet, C. Jourdain-Annequin (éd.), Héraclès: d'une rive à l'autre de la Méditerranée: bilan et perspectives, Brussels - Rome 1992.

BowIE 2014: E. Bowie, «Stesichorus’ Geryoneis», in L. Breglia, A. Moleti (eds), Hespería: tradizioni, rotte, paesaggi, Paestum 1914, 99-104.

BREGLIA 2013: L. Breglia, «Titani, Cureti, Eracle. Mitopoiesi euboica e guerra lelantina», in C. Bearzot, F. Landucci (ed.), Tra mare e continente. L'isola Eubea, Milano 2013, 17-65.

BRIQUEL 1999: D. Briquel, «La référence à Héraklès. De part et d'autre de la révolution de 509», in F.-H. Massa-Pairrault (dir.), Le mythe grec dans l'Italie antique. Fonction et image. Actes du colloque international organisé par l'École française de Rome, l'Istituto italiano per gli studi filosofici (Napoli) et l'UMR 126 CNRS (Archéologies d'Orient et d'Occident), Rome, 14-16 novembre 1996, Rome 1999, 101-120.

BTCGI: G. Nenci, G. Vallet (eds.), Bibliografia Topografica della Colonizzazione Greca in Italia e nelle isole tirreniche, I-XXII, Pisa-Roma 1982-2013.

CAMASSA 1987: G. Camassa, «I culti dell"area dello Stretto», in Lo Stretto crocevia di culture, Atti XXVI Convegno di Studi sulla Magna Grecia, Taranto-Reggio Calabria, 9-14 ottobre 1986, Taranto 1987, 133-162.

CAPDEVILLE 1999: G. Capdeville, «Heraclès et ses hôtes», in F.-H. Massa-Pairrault (dir.), Le mythe grec dans l'Italie antique. Fonction et image. Actes du colloque international organisé par l'École 
française de Rome, l'Istituto italiano per gli studi filosofici (Napoli) et l'UMR 126 CNRS

(Archéologies d'Orient et d'Occident), Rome, 14-16 novembre 1996, Rome 1999, 29-99.

CERCHIAI 1997: L. Cerchiai, «Intervento», in Mito e storia in Magna Grecia, Atti XXXVI Convegno di Studi sulla Magna Grecia, Taranto-Reggio Calabria, 9-14 ottobre 1996, Taranto 1997, 125-130.

CGO III: A.M. Biraschi, M. Cipriani, G. Greco, A. Taliercio Mensitieri, Culti greci in Occidente, III. Poseidonia-Paestum, Taranto 2013.

CiPRIANi, Pontrandolfo 2012: M. Cipriani, A. Pontrandolfo, «Mobilità e dinamiche insediative nel Golfo di Salerno», in Alle origini della Magna Grecia. Atti L Convegno di Studi sulla Magna Grecia, Taranto, 1-4 ottobre 2010, Taranto 2012, 989-1015.

COARELLI 1988: F. Coarelli, Il Foro Boario, Roma 1988.

Coppola 2001: A. Coppola, «Mito e propaganda alla corte dionisiana», in N. Bonacasa, L. Braccesi, E. De Miro, La Sicilia dei due Dionisî, Atti della settimana di studi, Agrigento 24-28 febbraio 1999, 371-386.

CORDANO 2014: F. Cordano, «Un periplo del Mediterraneo con le vacche di Gerione», in L. Breglia, A. Moleti (ed.), Hespería. Tradizioni, rotte, paesaggi, Paestum 2014, 137-146.

D'Agostino 1995: B. d'Agostino, Eracle e Gerione. La struttura del mito e la storia, Annali dell'Istituto universitario Orientale di Napoli (archeol) 2 (1995), 7-13.

D’Agostino 1997: B. d'Agostino, «Intervento», in Mito e storia in Magna Grecia, Atti XXXVI Convegno di Studi sulla Magna Grecia, Taranto-Reggio Calabria, 9-14 ottobre 1996, Taranto 1997, 222-223.

D'AMORE 2007: L. D'Amore, Iscrizioni Greche d'Italia. Reggio Calabria, Roma 2007.

DE LA GENIÈRE 1999: J. de La Genière, «Essai sur les véhicules de la légende d'Heraclès en Occident, in F.-H. Massa-Pairrault (dir.), Le mythe grec dans l'Italie antique. Fonction et image. Actes du colloque international organisé par l'École française de Rome, l'Istituto italiano per gli studi filosofici (Napoli) et l'UMR 126 CNRS (Archéologies d'Orient et d'Occident), Rome, 14-16 novembre 1996, Rome 1999, 11-27.

DE SENSI SESTITO 1982 : G. De Sensi Sestito, «Il santuario del Lacinio nella Lega achea e italiota», Miscellanea di Studi Storici, 2 (1982), 13-33.

DETIENNE 1960: M. Detienne, «Héraclès, héros pythagoricien», Revue de l'histoire des religions 158, 1 (1960), 19-53.

DuBoIS 2002: L. Dubois, Inscriptions grecques dialectales de Grande Grèce. II Colonies acheennes, Geneve 2002

EISENFELD 2018: H. Eisenfeld, «Geryon the Hero, Herakles the God», Journal of Hellenic Studies 138 (2018), 80-99.

FiCUCIELlo 2014: L. Ficuciello, «Il santuario ipogeico», in C. Rescigno, F. Sirano, Immaginando città. Racconti di fondazioni mitiche, forma e funzione delle città campane, Catalogo della mostra S. Maria Capua Vetere - Paestum, Benevento-Caserta 2014, 248-53.

FRANZEN 2009: C. Franzen, «Sympathizing with the monster: making sense of colonization in Stesichorus Geryoneis», Quaderni Urbinati di Cultura Classica 92 (2009), 55-72.

FRISONE 2011: F. Frisone, «Le lamentazioni dei Poseidoniati, (Aristox., fr. 124 Wehrli): culto eroico e memorie identitarie della comunità», in M. Lombardo-C. Marangio (eds.) Antiquitas. Studi di antichità in onore di Salvatore Alessandrì, Galatina 2011, 77-90. 
FRISONE 2014: F. Frisone, «Sulle tracce del Periegeta. Prospettive, contatti e interazioni nella descrizione dell'Illiria meridionale nelle fonti geografiche greche», in G.Tagliamonte (ed.), Ricerche archeologiche in Albania, Roma 2014, 81-102.

FRISONE 2017: F. Frisone, «“Tirando il dio per la giacchetta...”. Eracle e la Sicilia antica fra Calcidesi, Dori e altri», in M. Congiu, C. Miccichè, S. Modeo, Eracle in Sicilia. Oltre il mito: arte, storia, archeologia. Atti del XIII Convegno di Studi sulla Sicilia antica, Caltanissetta 2017, 137-167.

GENOVESE 1999-2000: G. Genovese, «Considerazioni sul culto di Herakles nella Calabria antica», Archeologia Classica n.s., I (1999-2000), 329-359.

GIANGIULIO 1983: M. GIANGIULIO, «Greci e non Greci in Sicilia alla luce dei culti e delle leggende di Eracle», in G.Nenci, G. Vallet, Modes de contacts et processus de transformation dans les sociétés anciennes, Pisa-Rome 1983, 785-846.

GIANGIULIO 1989: M. Giangiulio, Ricerche su Crotone arcaica, Pisa 1989.

GiANGIULIO 1996a: M. Giangiulio, «Le héros fondateur, l'espace sacrè de la déesse. Notes sur Hérakles et les santuaires d'Héra du Péloponnèse à la Grande Grèce», in C. Jourdain-Annequin, C. Bonnet (éd.), Héraclès, les femmes e le féminin. II rencontre héracléenne. Actes du colloque de Grenoble, 22-23 octobre 1992, Bruxelles-Rome 1996, 215-33.

GIANGIULIO 1996b: M. Giangiulio, «Tra mare e terra: l'orizzonte religioso del paesaggio costiero», in F. Prontera (a cura di), La Magna Grecia e il mare: studi di storia marittima, Taranto 1996, 251-271. GIANGIULIO 2000: M. Giangiulio, Pitagora. Le opere e le testimonianze. I-II, Milano 2000.

GIANGIULIO 2002: M. Giangiulio, «I culti delle colonie achee d'Occidente. Strutture religiose e matrici metropolitane», in E. GRECO, Gli Achei e l'identità etnica degli Achei d'Occidente (Atti del Convegno Internazionale di studi, Paestum (Salerno), 23-25 febbraio 2001) (Tekmeria 3) Paestum/ Atene 2002, 283-313.

GiANGIULIO 2011: M. Giangiulio, «Dioniso in Magna Grecia. Tradizioni letterarie e documenti epigrafici», in Atti IL Convegno Internazionale di Studi sulla Magna Grecia La vigna di Dioniso, vite, vino e culti in Magna Grecia, Taranto 24-28 settembre 2009, Taranto 2011, 429-445.

GiAnNell 1963: G. Giannelli, Culti e miti della Magna Grecia - Contributo alla storia più antica delle colonie greche in Occidente, Firenze [1924] 1963.

GIULIANI 1999: L. Giuliani, «Contenuto narrativo e significato allegorico nell'iconografia della ceramica apula», in F. de Angelis, S. Muth (hrsg von), Im Spiegel des Mythos. Bilderwelt und Lebenswelt/Lo specchio del mito. Immaginario e realtà. Atti del Colloquio (Roma 19-20 febbraio 1998), Wiesbaden 1999, 43-52.

GRECO 1998: E. Greco, «Agora eumeghetes. L'espace public dans les poleis d'Occident», in F. de Polignac, P. Schmitt Pantell (edd.), Public et privé en Grèce ancienne: lieux, conduites, pratique. Colloque organisé par le Centre L. Gernet, Ktema 24 (1998), 153-158.

GRECO 2009: E. Greco, «Le esperienze coloniali greche, modelli e revisioni. Introduzione ai lavori», in M. Lombardo, F. Frisone (eds) 2009: Colonie di colonie. Le fondazioni sub-coloniali greche tra colonizzazione e colonialismo, Galatina 2009, 9-16.

GreCo, Theodorescu 1983: E. Greco, D. Theodorescu (edd.), Poseidonia-Paestum. II. L'Agora, Rome 1983. HN 3: K.Rutter (principal ed.), Historia Numorum. Italy, London 2003.

HN 1911: B.V. Head, Historia Numorum. A Manual of Greek Numismatics, Oxford $1911^{2}$ 
HOLT 1992: P.Holt, «Herakles' Apotheosis in Lost Greek Literature and Art», L'antiquité classique 61 (1992), 38-59.

INTRIERI 2011: M. Intrieri, «Corcira fra Corinto e l'Occidente: rapporti e sincronismi di colonizzazione», in G. De Sensi Sestito, M. Intrieri, Sulla rotta per la Sicilia: L'Epiro, Corcira e l'Occidente, Pisa 2011, 175-208.

INVENTORY 2004: M. H. Hansen, T. Heine Nielsen (eds.), An Inventory of Archaic and Classical Poleis, Oxford 2004.

Iozzo 1996: M. Iozzo, «La ceramica "calcidese”. Temperie artistica e produzione artigianale a Rhegion in età arcaica», in E. Lippolis (ed.), I Greci in Occidente. Arte e artigianato in Magna Grecia, Catalogo della Mostra Taranto 1996, 313-321.

JEFFERY 1961: L.M. Jeffery, The Local Scripts of Archaic Greece, Oxford 19612.

JOURDAIN-ANNEQUIN 1982: C. Jourdain-Annequin, Héraclès en Occident. Mythe et histoire, Dialogues d'Histoire Ancienne 8 (1982), 227- 282.

JOURDAIN-ANNEQUIN 1989: C. Jourdain-Annequin, Héraclès aux portes du soir: mythe et histoire, Paris 1989.

JOURDAIN-ANNEQUIN 1992a: C. Jourdain-Annequin, «Héraclès en Occident», in C. Bonnet, C. JourdainAnnequin (éd.), Héraclès: d'une rive à l'autre de la Méditerranée: bilan et perspectives, Brussels - Rome 1992, 263-291.

JOURDAIN-ANNEQUIN 1992b: C. Jourdain-Annequin, «A propos d'un rituel pour Iolaos à Agyrion. Héraclès et l'initiation des jeunes gens», in A. Moreau (éd.) L'initiation. Actes du colloque international (Montpellier 11-14 avril 1991), Montpellier 1992,121-141.

LANE FoX 2008: R. Lane Fox, Travelling Heroes: Greeks and their Myths in the Epic Age of Homer, London 2008.

LLOYD-JONES 1980: H. Lloyd-Jones, «Stesicoro», in L'epos greco in Occidente, Atti XIX Convegno di Studi sulla Magna Grecia, Taranto 7-12 Ottobre 1979, Taranto1980, 9-28.

LONGO 2016: F. Longo, «Alcune riflessioni sullo spazio pubblico nelle poleis della Magna Grecia», in Poleis e politeiai nella Magna Grecia arcaica e classica, Atti LIII Convegno Internazionale di Studi sulla Magna Grecia, Taranto 27-30.

MALKIN 1994: I. Malkin, Myth and Territory in the Spartan Mediterranean, Cambridge 1994.

MASSERIA, TORELLI 1999: C. Masseria, M. Torelli, «Il mito all'alba di una colonia greca. Il programma figurativo delle metope dell'Heraion alla foce del Sele», in F.-H. Massa-Pairrault (dir.), Le mythe grec dans l'Italie antique. Fonction et image. Actes du colloque international organisé par l'École française de Rome, l'Istituto italiano per gli studi filosofici (Napoli) et l'UMR 126 CNRS (Archéologies d'Orient et d'Occident), Rome, 14-16 novembre 1996, Rome 1999, 205-262.

MeLE 1986: A. Mele, «Crotone e la sua storia», in Crotone. Atti XXIII Convegno di Studi sulla Magna Grecia, Taranto 1-10 ottobre 1983, Taranto1986, 9-87.

MELE 1997: A. Mele, «Il processo di storicizzazione dei miti», in Mito e storia in Magna Grecia. Atti del XXXVI Convegno di Studi sulla Magna Grecia, Taranto 4-7 ottobre 1996, Taranto1997, 152-166.

Mele 2009: A. Mele, «Cuma Opicia tra Greci e Romani», in Cuma. Atti XLVIII Convegno di Studi sulla Magna Grecia (Taranto 27 settembre-1 ottobre 2008), Taranto 2009, 77-167.

MERIANI 2003: A. Meriani, Sulla musica greca antica. Studi e ricerche, Napoli 2003. 
MinGAZZINI 1925: P. Mingazzini, «Le rappresentazioni vascolari del mito dell'apoteosi di Herakles», in Atti della Reale Accademia Nazionale dei Lincei, Memorie della classe di scienze morali, storiche, $e$ filologiche s. 6, 1 (1925), 413-490.

MURolo 1995: N. Murolo, «Le saline herculeae di Pompei. Produzione del sale e culto di Ercole nella Campania antica», in Studi sulla Campania preromana, Roma 1995, 103-125.

NAFISSI 1997a: M. Nafissi, «Riso fatale. Herakles e Kalchas a Herakleia Lucana (Lyk., Alex.979-81; schol. vet. 978; 980)», La Parola del Passato LII (1997), 30-62.

NAFISSI 1997b: M. Nafissi, «Rapporti fra le poleis e dinamiche interne nelle tradizioni miticostoriche: Siris-Eraclea e Taranto», in Mito e storia in Magna Grecia, Atti XXXVI Convegno di Studi sulla Magna Grecia, Taranto-Reggio Calabria, 9-14 ottobre 1996, Taranto 1997, 305-322.

NAFISSI 1997C: M. Nafissi, «Atene e Metaponto: ancora sulla Melanippe Desmotis e i Neleidi», Ostraka, 6, 2 (1997), 337-356.

PAGE 1973: D. L. Page, «Stesichorus: the Geryoneis», Journal of Hellenic Studies 93 (1973), 136-54.

Prestianni Giallombardo 2017: A. M. Prestianni Giallombardo, «Eracle sulle opposte sponde dello Stretto di Messina», in M. Congiu, C. Miccichè, S. Modeo, Eracle in Sicilia. Oltre il mito: arte, storia, archeologia. Atti del XIII Convegno di Studi sulla Sicilia antica, Caltanissetta 2017, 69-102.

SESTIERI 1955: P.C. Sestieri, «Il sacello poseidoniate», Bollettino d'Arte 2 (1955), 53-64.

SHAPIRO 1983: H. A. Shapiro, "Hêrôs Theos": The Death and Apotheosis of Herakles», The Classical World 77, 1 (1983), 7-18.

STAFFORD 2010: E. Stafford, «Herakles between gods and heroes», in J. Bremmer, A. Erskine (eds), The Gods of Ancient Greece: Identities and Transformations, Edinburgh 2010, 228-244.

STAFFORD 2012: E. Stafford, Herakles, London and New York.

StAZIO 1986: A. Stazio, «Problemi della monetazione di Crotone», in Crotone. Atti XXIII Convegno di Studi sulla Magna Grecia, Taranto 1-10 ottobre 1983, Taranto1986, 369-398.

TALIERCIO MENSITIERI 1997: M. Taliercio Mensitieri, «Intervento», in Mito e storia in Magna Grecia, Atti XXXVI Convegno di Studi sulla Magna Grecia, Taranto-Reggio Calabria, 9-14 ottobre 1996, Taranto 1997, 357-65.

TORELLI 1993: M. Torelli, «Gli aromi e il sale. Afrodite ed Eracle nell'emporia arcaica dell'Italia», in A. Mastrocinque (ed.), Ercole in Occidente. Atti del Convegno, Trento 1990, Trento 1993, 91-117.

VALENZA MELE 1979: N. Valenza Mele, «Eracle euboico a Cuma. La gigantomachia e la via Heraclea», in Recherches sur les cultes grecs et l'Occident, I, Naples 1979, 19-51.

VERBANCK-PIÉRARD 1987: A.Verbanck-Piérard, «Images et croyances en Grèce ancienne : représentations de l'apothéose d'Héraklés au VIe siècle», in C. Bérard (éd) Images et sociétés en Grèce ancienne, Lausanne 1987, 187-199.

VIAN 1952: F. Vian, La guerre des Géants, le mythe avant l'époque hellénistique, Paris 1952.

WONTERGHEM 1992: F. van Wonterghem, «Il culto di Ercole fra i popoli osco-sabellici», in C. Bonnet, C. Jourdain-Annequin (éd.), Héraclès: d'une rive à l'autre de la Méditerranée: bilan et perspectives, Brussels - Rome 1992, 319-351.

WONTERGHEM 1998: F. van Wonterghem, «Hercule et les troupeaux en Italie centrale : une nouvelle mise au point», in C. Bonnet, C. Jourdain-Annequin, V. Pirenne-Delforge (éd.), Le Bestiaire d'Héraclès: IIIe Rencontre Héracléenne, Liège 1998, 241-255. 
ZANCANI MONTUORO 1954: P. Zancani Montuoro, «Il Poseidonion di Poseidonia», Archivio Storico per la Calabria e la Lucania, 1954, 165-185.

ZANCANI MonTUORO1964: P. Zancani Montuoro, «Heraion alla foce del Sele, I. Altre metope del 'Primo thesauros'», Atti e Memorie Società Magna Grecia n.s. 5 (1964), 57-95.

ZANCANI MONTUORO-ZANOTTI BIANCO 1954: P. Zancani Montuoro, U. Zanotti Bianco, F. Krauss, Heraion alla foce del Sele, II, Roma 1954.

\section{NOTE}

1. Vd., fra la vasta letteratura in merito, i capisaldi: B AYET 1926; J OURDAIN-ANNEQUIN 1982; GiANGIULIO 1983; CAPDEVILLE 1999; DE LA GeNIERE 1999; MASSERIA-TORELli 1999; MALKIN 1994.

2. GIANNELLI 1963 ma accanto a questo anche BAYET 1926.

3. GIANGIULIO 1989, 67-79, in part. 70-2; GIANGIULIO 1996a; GIANGIULIO 2002, 286-7.

4. GiangiUlio 1989, 70-2, 74 per la cit..

5. Sulle metope dell'Heraion del Sele si veda almeno ZANCANI-ZANOTTI 1954, con ZANCANi 1964 e la minuziosa analisi interpretativa in MASSERIA, TORELLI 1999. Per una visione complessiva della documentazione e delle interpretazioni vd. ora M. Cipriani in CGO III, , con bibliografia precedente. Per la presenza di altre testimonianze sulle decorazioni architettoniche in luoghi di culto vd. i frammenti di un fregio fittile della prima metà del VI sec., pertinente alla decorazione di un edificio, da S. Mauro di Corigliano Calabro, nella parte interna della piana di Sibari, interpretati dal De Franciscis come scena di lotta fra Eracle e Nereo: GENOVESE 1999-2000, 336.

6. MASSERIA, TORELLI 1999, 249-53.

7. SHAPIRO 1983, 8. Vd. un ipotetico catalogo di possibili rielaborazioni delle vicende mitiche riguardanti l'eroe in opere letterarie e figurative di età arcaica e classica in HolT 1992.

8. Vd. in gen. BOARDMAN ET ALII 1988; 1990. Per quanto riguarda specifiche rielaborazioni figurative di temi della saga di Eracle che si possano attribuire all'ambiente magnogreco vd. l'ipotesi che l'officina Pittore delle Iscrizioni sia da localizzare a Rhegion: D’Agostino 1995 e Iozzo 1996. Per il repertorio della scultura architettonica vd. MASSERIA, TORELLI 1999.

9. FRISONE 2017, 139-43.

10. Tema che ha sempre con maggiore frequenza attirato l'attenzione degli studiosi, a partire dall'ancor prezioso studio di BAYET 1926, la diffusione del culto di Eracle fra i popoli dell'Italia antica inquadra contesti diversi e canali differenti, che vanno dai contatti emporici (ToReLli 1993), in cui un'importanza centrale hanno le saline e le vie del sale (COARell 1988; MURoLo 1995), in rapporto, a loro volta, con la transumanza e l'allevamento, al radicamento fra le popolazioni pastorali delle regioni appenniniche (WONTERGHEM 1992; 1998) o tirreniche (C ERCHIAI 1997). Momento cruciale appare, nella Roma della fine del VI secolo, l'elaborazione di vere strategie di rappresentazione, in chiave mitico-genealogica eracleica, strettamente collegate a quelle sviluppatesi nel mondo greco e che rivestono una chiara funzione in ambito pienamente politico, pur rispondendo a differenti istanze e posizioni: BRIQUEL 1999; AMPOLO 2017.

11. BONNET 1988; BONNET-JOURDAIN-ANNEQUIN 1992; BONNET 2005 e in gen. BERNARDINI, ZUCCA 2005.

12. Certo impossibile da definire esaustivo, il quadro delle evidenze archeologiche e documentarie è sicuramente ormai ben più ampio dell'epoca in cui se ne tentavano le prime sintesi: vd. infatti ora il progetto organico di pubblicazione della documentazione relativa ai culti delle poleis magnogreche (Culti greci in Occidente: CGO) del quale si è giunti al III volume (Poseidonia, preceduto da quello di Taranto e di Eraclea). Sintetiche ma molto utili rassegne dei dati documentari anche relativi ai culti, sono, ss.vv., città per città, in BTCGI e in Inventory. 
13. Si tratta dell'articolo «Eracle in Magna Grecia, fra miti, riti e diplomazia», che verrà pubblicato sul prossimo numero di Mythos, Ad esso si dovrà fare riferimento a più riprese nel corso del presente lavoro.

14. BONNET 2008, 345-6; 350-1.

15. Vd. Page 1973; Mele 1997, 153-60; Franzen 2009; BowIE 2014. Si considerino, in particolare, con D'AGOSTINO 1995, 7-9 e MELE 1997, 154-5, gli elementi che lasciano intravedere una precedenza del tema mitico del viaggio di Eracle verso la terra rossa del Sole (Erytheia) ove risiede Gerione alla localizzazione di questa in area iberico-tartessica, che è propria di Stesicoro (fr. 7 Page) e fa da premessa necessaria a molte delle saghe locali di Eracle in Magna Grecia. Tracce di una diversa visione spaziale del mito p. es. in Ecateo (FGrHist $1 \mathrm{~F}$ 126), su cui vd. anche FRISONE 2014, 96-7. Su Stesicoro nel quadro della produzione epica dell'Occidente vd. LLOYD-JONES 1980. Per le tematiche eracleiche sviluppate dal rapsodo di Himera vd. in sintesi STAFFORD 2012 ,17, 42-50, 66, 68.

16. BONNET 2008, 342.

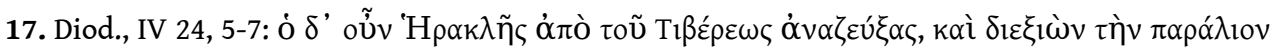

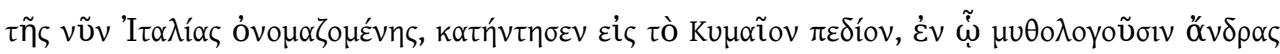

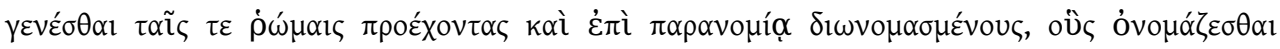

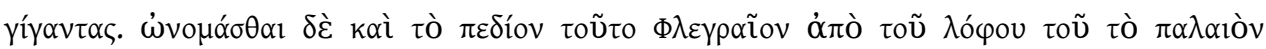

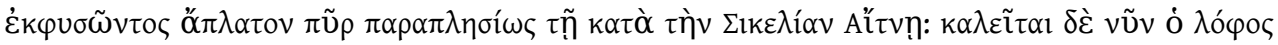

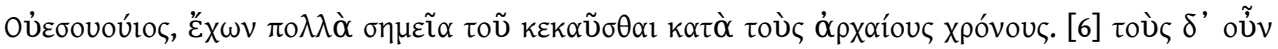

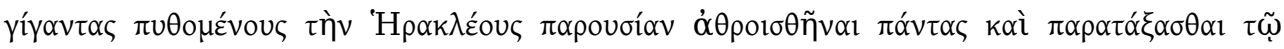

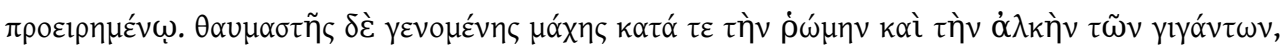

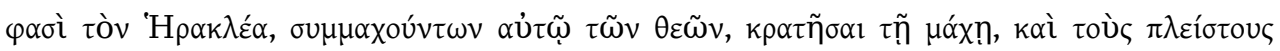

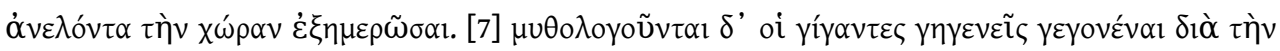

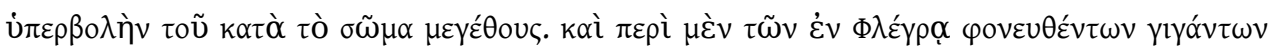

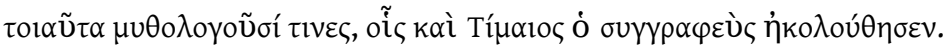

18. Cfr. Timaeus, FGrHist 566 F 89.

19. La battaglia fra dei e Giganti (VIAN 1952), plasmata sul mito esiodeo della lotta fra dei olimpici e Titani (Hes., Theog. 617-720), è tradizionalmente fatta svolgere a Phlegra (Aesch., Eum. 295; Pind. Nem. I, 67-8; Isth. 6, 33-34; Aristh., Av.823-5). Pongono questa nella Pallene Hdt. VII, 123 ma anche Diod., IV, 15, 1; V, 71, 4. e Apollod. I, 6, 1. Presso Cuma Tim. FGrHist 566 F 89 apud, Diod. IV, 24, 7 e Diod. V, 71, 4.; Strabo, V, 4, 4, 6; Pol. III, 91, 7. Ma anche in Arcadia Paus. VIII, 29,1 o a Rodi Diod. $\mathrm{V}, 55,5$. Per il rilievo del tema mitico, in riferimento alla Titanomachia, in ambito euboico vd. BREGLIA 2013.

20. Vd., per la topografia mitica, i riferimenti raccolti in BTCGI, VII, s.v. Cuma.

21. ValenZa Mele 1979; LANe Fox 2008, 309-13; Mele 2009, 114-7.

22. Hellan. FGrHist 4 F 111 apud, D.H. I, 35; Tim. FGrHist 566 F 90 apud, Diod. IV, 22, 6; Ps Apollod. 2,5,10; Gell. 11,1,1; Paus, III, 16,5 CAMASSA 1987; GIANGIULIO 1996b; BONNET 2008, 346-47; FRISONE 2017, 156-60; Prestianni Giallombardo 2017.

23. GiangiUlio 1983, CAPDEVILLE 1999, 61-83. In questo segmento del viaggio di ritorno di Eracle, da un lato è stata notata la non fluida connessione con il percorso periegetico condotto fino alla Sicilia (CORDANO 2014, 142), dall'altro si giustappongono alla cornice itineraria miti che raccontano di una permanenza dell'eroe in luoghi specifici (il fiume Aleco, Crotone, Metaponto) in cui il suo soggiorno porta trasformazioni e benefici ai contesti naturali, come l'uccisione di mosche e cavallette, o il noto mito della scomparsa delle cicale (Diod., IV 22, 5): BAYET 1926, 401-4; BONNET 2008, 351.

24. Sezione «Eracle in Magna Grecia, fra miti, riti e diplomazia», pubblicato a seguire, vd. supra nota 13.

25. Vd. CAPDEVILLE 1999, 75-6; 82. 
26. Per il topos, vd. Giangiulio 1983, Jourdain-AnNeQuin 1989; 1992a; CERCHIAI 1997, 128-9; CAPDEVILle 1999.

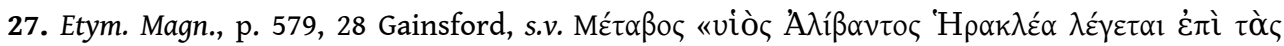

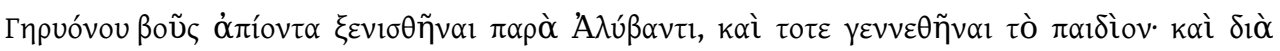

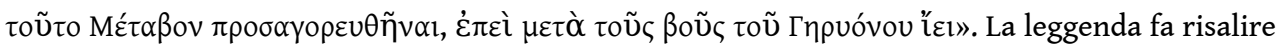
al passaggio di Eracle, qui accolto da Alibàs/Alybas, la spiegazione paretimologica del nome di Metabos, eroe eponimo di Metaponto. La testimonianza intorno ad un heroon di Metabos è in Antioco, FGrHist $555 \mathrm{~F}$ 11. Sulla rivalutazione della tradizione presente nella fonte lessicografica vd. NAFISSI 1997c, 353-356, che ritiene il gioco paretimologico essenziale presupposto alla notizia di Antioco e, più ancora, a una serie di indizi che legano a una fase di metà $\mathrm{V}$ secolo il culto dell'eponimo e le prime emissioni magnogreche con raffigurazione di Eracle, proprio a Metaponto: su ciò vd. più in dettaglio nella seconda parte di questo lavoro, dal titolo «Eracle in Magna Grecia, fra miti, riti e diplomazia», supra, nota 13.

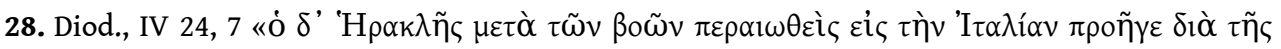

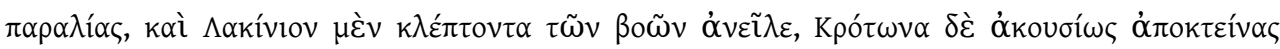

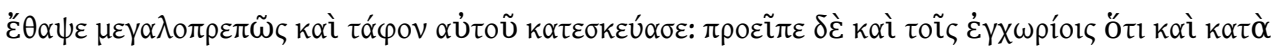

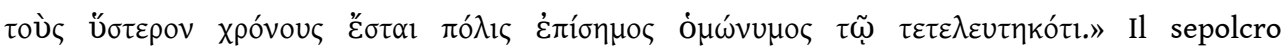
dell'eponimo Crotone è elemento costitutivo del paesaggio religioso di Crotone anche in Ov., Met. XV 53-57, fonte nella quale Eracle è visto se non fondatore, fautore della futura nascita della città (19-23), la cui fondazione da parte di Miscello è dovuta alla spinta del suo nume divino e compie la profezia che egli aveva pronunziato nel percorrere quei luoghi con la mandria gerionica (12-18). cf. Alcim. Sic. apud Ath. X, 441 a; Iambl. V. Pyth., IX, 50; Sil. Ital. XI 19; Serv. Ad Aen III, 552. Su queste fonti e su Ovidio in particolare CAPDEVILLE 1999, 61-69.

29. GianNelli 1963, 148; GiangiULio 1989, 68.

30. GianNelli 1963, 141 n. 4; De SENSI 1982, 31 e n. 95 (entrambi con riferimenti alla bibliografia prec.); GIANGIULIO 1989, 70-72, per il quale il rapporto Hera-Herakles risale alla veneranda tradizione cultuale del Lacinio, mentre Stazio 1986, 385; 1996, 270 attribuisce valore politico attualizzante (in chiave anti-ateniese e filo-spartana) a questo richiamo a una tradizione di cui caratteri arcaici della legenda starebbero a significare l'antichità.

31. Per la discussione dettagliata di queste e delle altre emissioni monetali magnogreche con riferimento a Eracle si rinvia all'altra parte di questa ricerca, pubblicata a seguire nell'articolo «Eracle in Magna Grecia, fra miti, riti e diplomazia» (vd. supra nota 13).

32. HN 1911, 96-100; RUTTER, HN 3, 2139.

33. JEFFERY 1961, 257.

34. Iambl., VPyth, 9, 50: vd infra. Secondo GIANGIULIO 2000, II, 530 si tratterebbe di tradizioni che nel loro nucleo essenziale, relativo ai destinatari delle prime predicazioni pitagoriche e Crotone, sono attestate già con Antistene e poi in Dicerarco. Nella loro definizione, e nel probabile arricchimento di contenuti, sembra riconosciuto il ruolo di Timeo (giunto a Giamblico via Apollonio di Tiana) che ricorre anche fra le fonti di Diodoro per i percorsi di Eracle nell'estrema Italia (FGrHist $556 \mathrm{~F}$ 90).

35. Serv. Ad Aen.3, 552 «Iunonis Laciniae templum, secundum quosdam a rege conditore dictum, secundum alios a latrone Lacino, quem illic Hercules occidit, et loco expiato, Iunoni templum constituit...Quidam dicunt templum hoc Iunonis a Lacinio rege appellatum, cui dabat superbiam mater Cyrene et Hercules fugatus; namque eum post Geryonem exstinctum de Hispania reuertentem hospitio dicitur recipere noluisse, et in titulum repulsionis eius templum Iunoni tanquam nouercae, cuius odio Hercules laborabat, condidisse.» Giangiulio 1989, 70-71 e nota 77 considera di ascendenza timaica anche questa tradizione.

36. Rutter, HN 3 2159. Si tratta delle emissioni di stateri argentei databili al periodo successivo al 400 a. C. (STAZIo 1986, 390), in cui alla figura di Eracle stante, rappresentato sul D/ con 
un'iconografia che si distingue da quella della precedente coniazione degli anni Venti, viene associata, sul R/, la testa di Hera Lacinia. Valorizzata già da DE SENSI 1982, 31 e n. 95, l'emissione è considerata di forte valore politico (STAZIO 1986, 390-94) in quanto farebbe riferimento alla centralità di Crotone e del santuario Lacinio per la Lega Italiota. In tal senso la ripresa del tipo presso altre poleis aderenti alla Lega italiota su cui vd. p.es. TALIERCIO MENSITIERI 1997, 358-59.

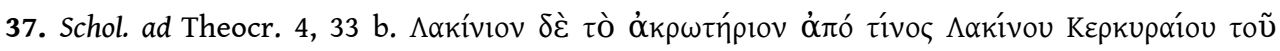
ن்

38. La similitudine dei racconti ha fatto pensare a confusioni ed errori nelle riprese seriori, soprattutto per la narrazione di Conone relativa a Locri, di cui è testimone il tardo Fozio: vd. CAPDEVILLE 1999, 75 e n. 221-222 con riferimenti alla bibliografia precedente e più di recente INTRIERI 2011, 194-196.

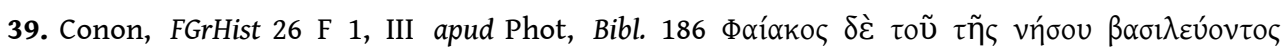

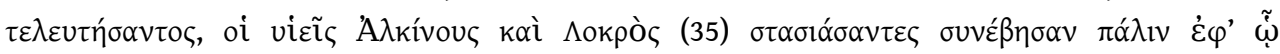

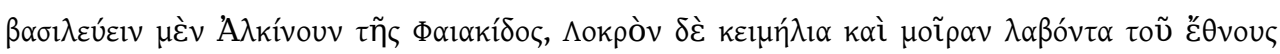

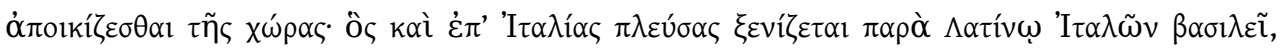

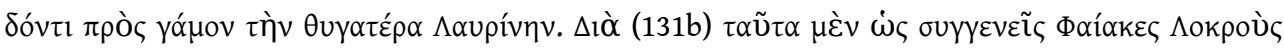

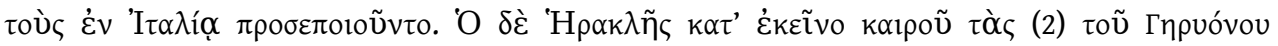

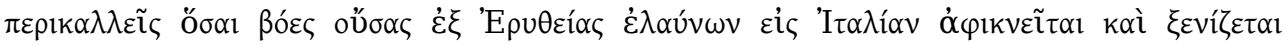

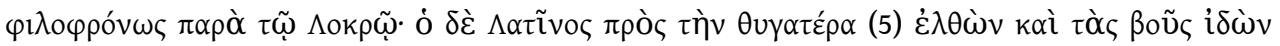

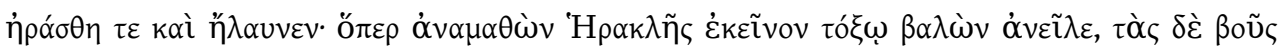

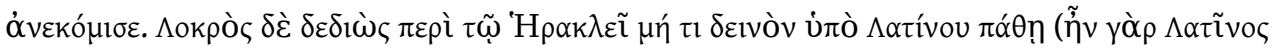

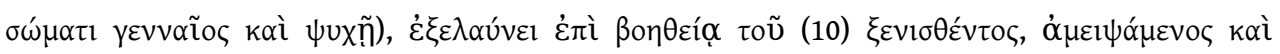

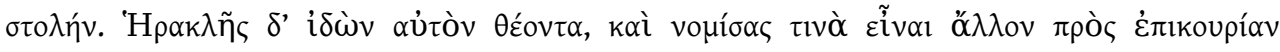

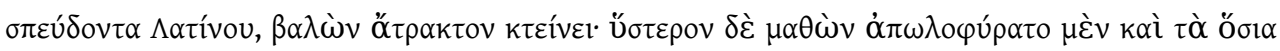

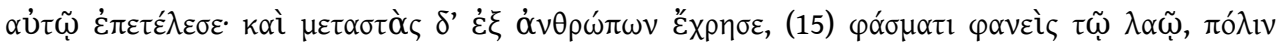

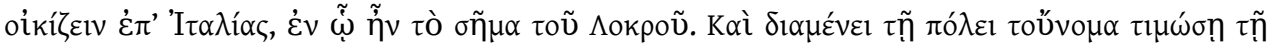

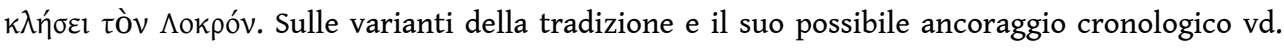
COPPOLA 2001, 376; INTRIERI 2011, 193-202.

40. CAPDEVILLE 1999, 75. Vd. ora NAFISSI 1997b, 320-1 per il significato e l'inquadramento più corretto da dare a quest'idea di "propaganda" attraverso il discorso mitico.

41. NAFISSI 1997a, 58.

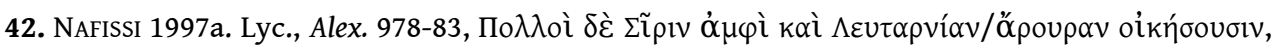

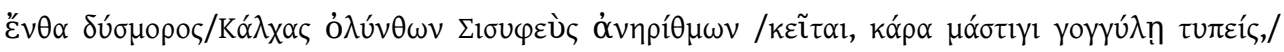

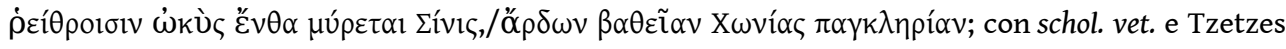
ad $978,980,1047$.

43. NAFISSI 1997a, 59.

44. Diod. IV, 24. Per i rituali di tipo iniziatico connessi al culto di Eracle vd. JoURDAIN-ANNEQUIN $1992 b$.

45. D’Agostino 1997, 223.

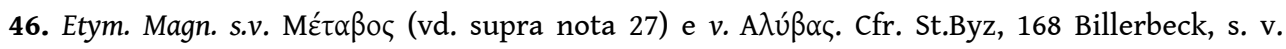

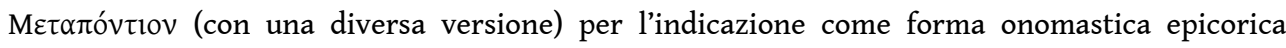
"barbara": cfr. NAFISSI 1997c, 344 nota 53; 353 nota 110.

47. La struttura, realizzata in blocchi di calcare, è costituita da un ambiente a camera $(2,56 \times 3,95)$ con copertura a doppio spiovente orientata perfettamente E/O. All'interno dell'ambiente, allestito con due banchine parallele sui lati lunghi, fra le quali era sistemata la tavola di legno, era disposto il corredo di oggetti pregiati databili fra il 540 e il 520 a.C. Essi costituiscono riferimento cronologico che consente di datare l'edifcio nell'ultimo venennio del VI secolo, mentre il materiale all'esterno e gli apprestamenti che inglobavano la piccola costruzione 
indicano differenti sistemazioni in più fasi successive, fino alla ridefinizione dell'agorà per dar luogo all'assetto del Foro della colonia latina sorta sull'antica città achea e poi lucana. Per una dettagliata descrizione della struttura e una sintesi delle fasi costruttive vd. FICUCIELLo 2014.

48. CIPRIANI, PONTRANDOLFO 2012, 1005.

49. GRECO 1998; LONGO 2016, 384-389, con rif. bibl.

50. GRECO 2009, 14. Quanto alle pratiche culto, MERIANI 2003 collega il contesto archeologico alla famosa testimonianza di Aristox., fr. 124 Wehrli, ritenendo che si riferisca a un culto funerario per l'ecista; contra FRISONE 2011. Vd. inoltre in CGO III: CIPRIANI, 113-4; BIRASCHI, 332-4; SESTIERI 1955; GRECO, THEODORESCU 1983, 25-33, 74-79.1.

51. Il legno di cipresso era essenza dal significato simbolico altissimo, della quale era fatto lo scettro di Zeus: da ciò, ad es. il tabù pitagorico che impediva di utilizzarlo per le bare, i roghi e le sepolture Iambl, V. Pyth., XXVIII, 155.

52. Vd. FiCUCIELLO 2014, 253, cat. 2.5.9.

53. SESTIERI 1955, 55 ricostruzione poi smentita dai dati di scavo: GRECO, THEODORESCU 1983, 24-28. Per l'iscrizione retrograda su olpe a f.n., una dedica alla ninfa: ARENA 1996, 50-51 = DUBOIS 2002, n. 24.

54. GRECO, THEODORESCU 1983, 74.

55. ZANCANi MONTUORO 1954, 183-85 suggeriva Is/Ois, ecista di Sibari, che sarebbe ivi onorato nel momento dell'innesto nella colonia sibarita di un consistente gruppo metropolitano; Ficuciello, associando alle evidenze epigrafiche graffite su ceramica (coppe ioniche con segno $\mathrm{M}$ ) il nome abbreviato (Megyl[los/Megyl[lias/Megyl[os) presente su alcune emissioni poseidoniati, formula l'ipotesi che sia questa la denominazione del "vero" ecista della città (vd. Ficuciello 2014). In sintesi, per l'idea di più fasi e di più soggetti coinvolti nella prassi del "culto per l'ecista" nel corso del tempo vd. GRECO 2009; CIPRIANI in CGO III, 114.

56. FICUCIELLO 2014, 248.

57. MINGAZZINI 1925, 419-36; BOARDMAN ET ALII 1990, 131.

58. E ISENFELD 2018, 82. Per l'elaborazione dell' apotheosis di Eracle nella poesia e nelle arti figurative vd. anche SHAPIRO 1983; VERBANCK-PIÉRARD 1987; HOLT 1992; STAFFORD 2010, 239-44.

59. Secondo ARDOVINO 1986, 34, che fra l'altro richiama proprio i casi di Crotone e Metaponto, sarebbe Eracle stesso a sovrapporsi all'ecista in qualità di fondatore.

60. DetienNe 1960, 23 mette in evidenza giustamente come il testo adombri l'esistenza di una tradizione orale indipendente e preesistente il discorso attribuito a Pitagora.

61. DETIENNE 1960, 23-24.

62. Diod. XII, 9, 5-6 A. Mordze in RE, XV, 2, 1932, coll.1672-6; DetienNe 1960; MeLE 1986, 21-2; GIANGIULIO 1989, 70; 298-300 e n. 27 e BTCGI, s.v. Crotone.

63. Hdt. I, 60. Per l'associazione fra l'episodio della storia politica di Atene, l'ideologia e la prassi arcaica di un personaggio come Pisistrato e la diffusione del tema iconografico dell'apotheosis di Eracle nella produzione ceramica attica a f.n. vd. BOARDMAN 1975; 1989. Una proiezione occidentale di questa associazione, con funzioni ideologiche per la rappresentazione del potere è stata riconosciuta nella Roma di fine VI secolo fra monarchia e repubblica (vd. BRIQUEL 1999) soprattutto in associazione all'interpretazione del gruppo statuario frontonale dell'area sacra di S. Omobono (su cui vd. da ultimo, ADORNATO 2003, per l'ampia bibliografia, e la disamina in AMPOLO $2017,75-78 ; 103-4$.). Un collegamento il questa direzione è stato suggerito anche per Aristodemo di Cuma: vd. MeLE 2009, 116-7.

64. DETIENNE 1960, 43-51.

65. Per il dionisismo italiota nella fase tardo arcaica e classica vd. GIANGIULIO 2011 e, in gen. il volume La vigna di Dioniso, vite, vino e culti in Magna Grecia, Atti del IL Convegno di Studi sulla Magna Grecia. 
66. GIULIANI 1999, 51.

67. È quanto possiamo dedurre dalle attestazioni dell'Eracle "Rhegino" della dedica epigrafica da Castellace (RC) (D'AMORE 2007, n. 57, 83-84), o da quelle lessicografiche del tarentino Eridantes

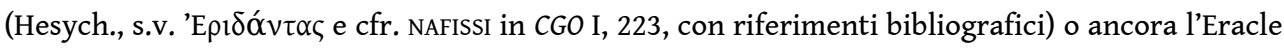
profeta-sacerdote dell'ambiente acheo, crotoniate e metapontino che emerge dalla documentazione numismatica (si rimanda ancora una volta al contributo "gemello" a questo, «Eracle in Magna Grecia, fra miti, riti e diplomazia», di p.p,: vd. supra nota 13) oltre che dalle tradizioni letterarie su cui infra. Vd. anche, per la Sicilia, FRISONE 2017.

68. APPADURAI 1996, 229-35.

\section{RIASSUNTI}

L'articolo presenta la prima parte di una più ampia ricerca dedicata alla presenza di Eracle nel panorama mitico-religioso e cultuale delle poleis di Magna Grecia. In questa sede si esaminano alcune tradizioni mitiche come pure in alcuni quadri documentari della Magna Grecia di piena età classica (ma forse già del tardo arcaismo) in cui Eracle si mostra come un agente attivo in alcuni rituali di eroizzazione. L'eroe-dio appare infatti una presenza importante e ricorrente i cui aspetti peculiari sono collegati ai processi di trasformazione/metamorfosi nella direzione del sacro. Egli rappresenta in tali contesti un fondamentale tramite - divino - che conduce altre figure allo status eroico, lasciando intravedere ideologie che riguardano la possibilità di una tale trasformazione e concreti processi di "eroizzazione", cui questa figura religiosa offre opportunità e strumenti, in termini religiosi e culturali.

The paper presents the first part of a wider study on Herakles' religious relevance both mythical traditions and documentary evidence concerning Greek cities of Magna Graecia. In this part some myths and material evidences will be investigated which show how in Magna Graecia, between the late 6th and the 5th centuries BC, Herakles becomes an active agent in some rituals of heroization. The hero-god appears indeed to be an important and recurrent presence in some regional myths and cults, whose peculiar aspects are connected to processes of change towards the heroic condition. Herakles represents here a fundamental - divine - agent which leads other figures to the heroic status. He gives opportunity and tools, in religious and cultural terms, to these actual processes, also hinting at ideologies that suggest the possibility of a transformation in the direction of the super-human.

\section{INDICE}

Keywords : Herakles, Magna Graecia, hero-cults, heroization, production of sacrality, production of locality-neighborhood

Parole chiave : Eracle; Magna Grecia, eroizzazione, produzione di sacralità, rapporti interpoleici 


\section{AUTORE}

\section{FLAVIA FRISONE}

Università del Salento

Dipartimento di Beni Culturali

Edificio ex INAPLI - Via Dalmazio Birago, 64

Lecce (LE)

flavia.frisone(at)unisalento.it 